3 World Health Organization. Global tuberculosis report 2013. Geneva, WHO Press, 2013.

4 Basu S, Stuckler D, Bitton A Projected effects of tobacco smoking on worldwide tuberculosis control: mathematical modelling analysis. BMJ 2011; 343: d5506.

5 Van Rie A, Patel MR, Nana M Integration and task-shifting for TB/HIV care and treatment in highly resource-scarce settings: one size may not fit all. J Acquir Immune Defic Syndr 2014; 65: e110-e117.

World Health Organization. Global status report on alcohol and health. Geneva, WHO Press, 2014.

Maruza M, Albuquerque MF, Coimbra I Risk factors for default from tuberculosis treatment in HIV-infected individuals in the state of Pernambuco, Brazil: a prospective cohort study. BMC Infect Dis 2011; 11: 351.

8 Kittikraisak W, Burapat C, Kaewsa-ard S Factors associated with tuberculosis treatment default among HIV-infected tuberculosis patients in Thailand. Trans R Soc Trop Med Hyg 2009; 103: 59-66.

9 Tabarsi P, Chitsaz E, Moradi A Treatment outcome, mortality and their predictors among HIV-associated tuberculosis patients. Int J STD AIDS 2012; 23: e1-e4.

10 Silva DR, Menegotto DM, Schulz LF Factors associated with mortality in hospitalized patients with newly diagnosed tuberculosis. Lung 2010; 188: 33-41.

\title{
Pulmonary hypertension associated with obliterative phlebitis in IgG4-related lung disease
}

\author{
To the Editor:
}

IgG4-related disease (IgG4-RD) is a systemic fibroinflammatory disease associated with elevated serum IgG4 level and IgG4-positive plasma cell infiltration into multiple organs [1]. In 2001, Hamano et al. [2] reported that patients with autoimmune pancreatitis have high-serum IgG4 concentrations. Subsequently, IgG4-related lesions similar to those of autoimmune pancreatitis have been identified in other organs [1] including the lungs [3].

Thoracic involvement in IgG4-RD has been increasingly recognised, usually manifesting as inflammatory pseudotumours, interstitial pneumonia or occasionally, pleuritis [3]. However, little information is available on airway disease or pulmonary hypertension $(\mathrm{PH})$ associated with IgG4-RD. In this report, we describe a patient presenting with $\mathrm{PH}$ associated with IgG4-related bronchiolitis.

A 45-year-old male first presented with community-acquired pneumonia caused by Haemophilus influenzae. When he was discharged, slight dyspnoea on exertion and abnormal findings in computed tomography (CT) of the chest remained. After 5 months of follow-up, he was admitted again for a close inspection of deteriorating dyspnoea, which was categorised as World Health Organization class II. He had a 10 -year history of refractory sinusitis. For the last 5 years, he had complained of swelling of the bilateral eyelids, which was diagnosed as "nonspecific dacryoadenitis" based on left lacrimal grand biopsy at a previous hospital clinic visit. He was an ex-smoker (one pack per day for 20 years) and had no relevant occupational or environmental exposures.

A chest CT scan manifested diffuse bilateral centrilobular nodules, prominent peripheral pulmonary vascular markings (fig. 1a) and mediastinal lymphadenopathy with no evidence of pulmonary large vessel compression (fig. 1b). Laboratory examinations showed high serum IgG $\left(6530 \mathrm{mg} \cdot \mathrm{dL}^{-1}\right)$ and $\operatorname{IgG} 4$ $\left(>1500 \mathrm{mg} \cdot \mathrm{dL}^{-1}\right.$ ) levels. Serum C-reactive protein, lactate dehydrogenase, Krebs von den Lungen-6 antigen and surfactant protein-D levels were normal. Results of evaluations for connective tissue diseases, angiotensin converting enzyme, human leukocyte antigen-B54 and human T-lymphotropic virus-1 antigen were all negative. Arterial blood gas showed normal partial pressure of oxygen and carbon dioxide (87.5 and 35.3 Torr, respectively). A pulmonary function test indicated obstructive disorder with decreased forced expiratory volume in $1 \mathrm{~s}$ (FEV1)/forced vital capacity ratio (FVC) and decreased predicted value for diffusing capacity of the lung for carbon monoxide (\%DLCO) (FEV1/FVC 67.3\%; \%DLCO 73.6\%). The predicted values for FVC (\%FVC) and FEV1 (\%FEV1) were normal (\%FVC 108.4\%; \%FEV1 81.6\%). Bronchoalveolar lavage fluid cell count was $0.32 \times 10^{5}$ cells $\cdot \mathrm{mL}^{-1}$, with a normal cellular profile and CD4/ CD8 ratio. Microbial culture results on the sputum and bronchoalveolar lavage fluid were negative. Although echocardiography disclosed no abnormalities, pulmonary arterial catheterisation revealed elevated pulmonary arterial pressure (PAP) (systolic $47 \mathrm{mmHg}$, diastolic $23 \mathrm{mmHg}$, mean $33 \mathrm{mmHg}$ ) 

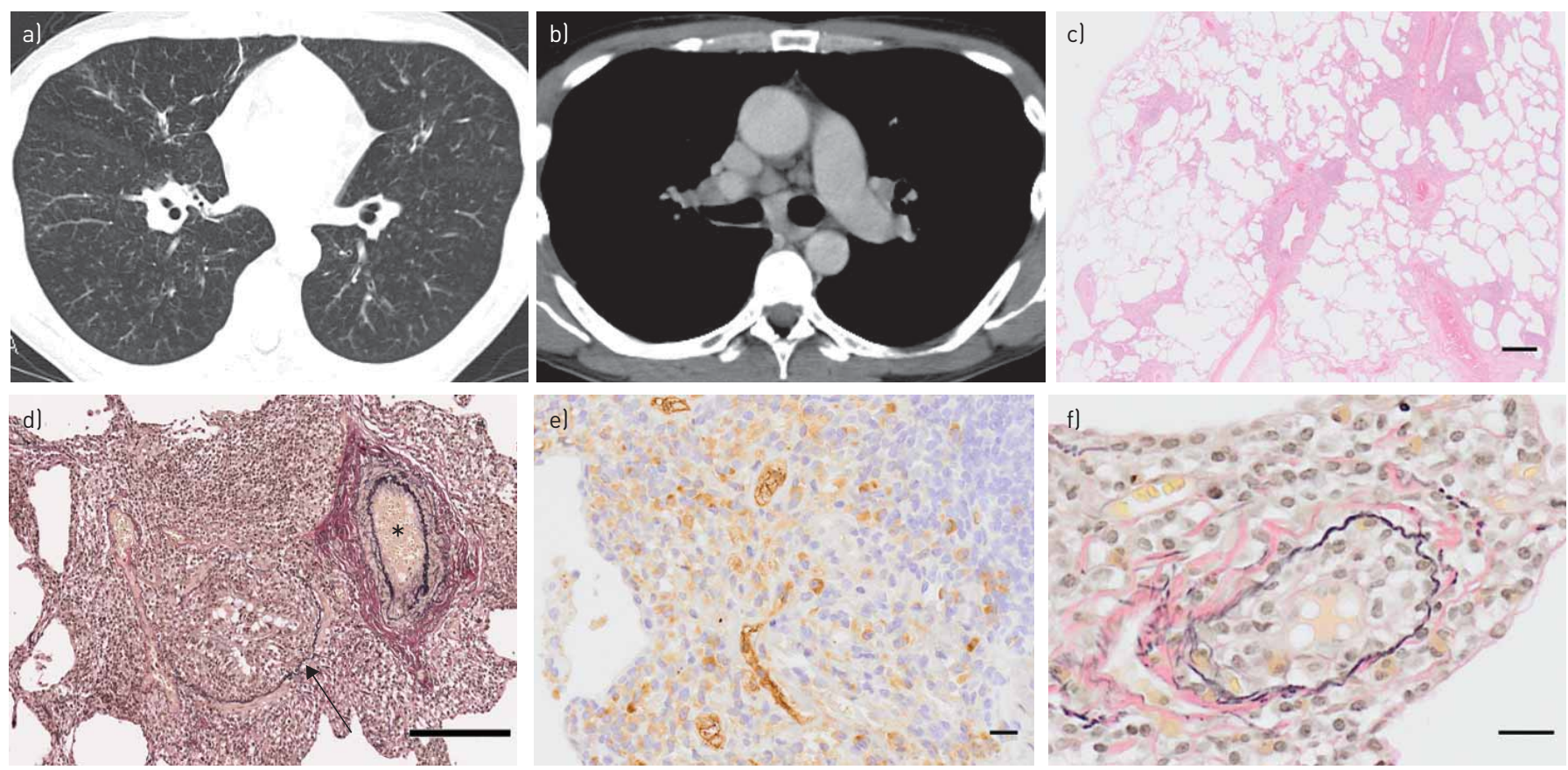

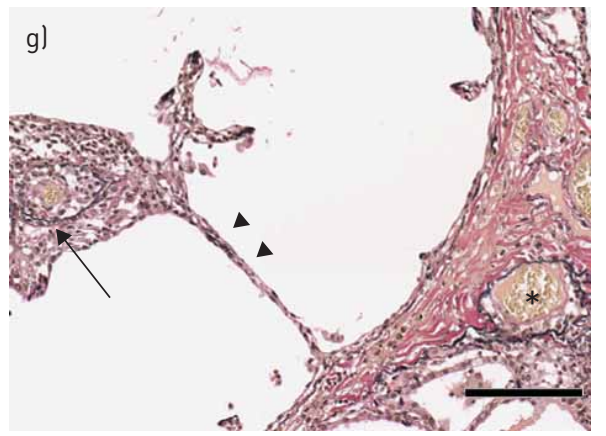

FIGURE 1 Radiological findings on chest computed tomography (CT) scan and histopathological findings in lung biopsy specimen. a) Chest CT scan showing diffuse bilateral centrilobular micronodules and prominent peripheral pulmonary vascular markings. b) Contrast-enhanced chest CT scan showing mediastinal lymphadenopathy with no evidence of pulmonary large vessel compression. c) Photomicrograph showing intense inflammation expanding from bronchioles into surrounding lung parenchyma (haematoxylin-eosin stain). d) Elastica van Gieson (EVG) stain showing constrictive bronchiolitis (arrow) and uninvolved artery (asterisk). e) IgG4 immunohistochemical stain showing a remarkably high number of IgG4-positive plasma cells per high-power field and an IgG4/IgG ratio of $\sim 50 \%$. f) EVG stain showing obliterative phlebitis with plasma cells. g) EVG stain showing obliterative phlebitis (arrow), uninvolved artery (asterisk) and adjacent alveolar septa with uninvolved capillaries (arrowheads). Scale bars: $500 \mu \mathrm{m}$ (c), $200 \mu \mathrm{m}$ (d and g) and $20 \mu \mathrm{m}$ (e and f).

and elevated pulmonary vascular resistance (PVR) (4.47 Wood units), with normal right atrial pressure (RAP) $(7 \mathrm{mmHg})$, cardiac index $\left(3.07 \mathrm{~L} \cdot \mathrm{min}^{-1} \cdot \mathrm{m}^{-2}\right)$ and pulmonary arterial wedge pressure (PAWP) ( $9 \mathrm{mmHg}$ ). 6-min walk distance (6MWD) was $648 \mathrm{~m}$, with $91 \%$ of minimum arterial oxygen saturation measured by pulse oximetry $(\mathrm{SpO} 2)$.

Suspecting IgG4-related lung disease, we performed video-assisted thoracoscopic surgery. The specimen exhibited marked peribronchiolar inflammation due to infiltrating plasma cells, lymphocytes and eosinophils. Some foci showed expanded infiltration into the surrounding lung parenchyma (fig. 1c and d), which corresponded with the diffuse bronchiolocentric nodules seen on chest CT. Immunostaining showed 30 IgG4-positive plasma cells per high-power field and an IgG4/IgG ratio of 50\% (fig. 1e). Nearly half of the medium-sized veins were involved through lymphoplasmacytosis, and a small number of veins among them showed destruction of the lamina elastica in their walls. These veins were considered to show obliterative phlebitis (fig. $1 \mathrm{~d}, \mathrm{f}$ and $\mathrm{g}$ ). No inflammation of the pleura and thrombus, and no veno-occlusive changes, capillary hemangiomatosis, capillaritis or obliterative arteritis were observed (fig. 1d and g). After confirming these findings, we obtained a lacrimal gland specimen from the previous hospital, in which notable infiltration of IgG4-positive plasma cells was observed with an IgG4/IgG ratio of $>50 \%$. Diagnoses of IgG4-related bronchiolitis and dacryoadenitis were made, and the cause of PH was guessed to be obliterative phlebitis. 
6 months after surgical biopsy, dyspnoea, eyelid swelling and postnasal discharge had worsened. \%FEV1 declined to 47\%. Right heart catheterisation showed elevated PAP (systolic $58 \mathrm{mmHg}$, diastolic $28 \mathrm{mmHg}$, mean $42 \mathrm{mmHg}$ ) and elevated PVR (5.18 Wood units) with normal RAP (8 $\mathrm{mmHg}$ ), cardiac index (3.41 L $\cdot \mathrm{min}^{-1} \cdot \mathrm{m}^{-2}$ ) and PAWP (11 mmHg). 6MWD decreased to $555 \mathrm{~m}$ with a minimum SpO2 of $85 \%$. Based on these findings, two cycles of methylprednisolone pulse $\left(1000 \mathrm{mg} \cdot \mathrm{day}^{-1}\right.$ for 3 days) were initiated, followed by $10 \mathrm{mg}$ of prednisolone and cyclosporin daily on the basis of the authors' experience with collagen tissue disease-related interstitial pneumonias [4]. This was associated with significant resolution of symptoms within 1 month without recurrence or any adverse events.

After 3 months of treatment, the abnormal CT findings had markedly improved. Serum IgG4 level decreased to $358 \mathrm{mg} \cdot \mathrm{dL}^{-1}$, while $\% \mathrm{FEV} 1$ and $\% D$ LCO increased to $89.0 \%$ and $96.9 \%$, respectively. A repeat pulmonary arterial catheterisation showed a decline in PAP (systolic $34 \mathrm{mmHg}$, diastolic $13 \mathrm{mmHg}, 23 \mathrm{mmHg}$ ) and a decline in PVR (2.30 Wood units) with normal RAP $(3 \mathrm{mmHg})$, cardiac index $\left(4.58 \mathrm{~L} \cdot \mathrm{min}^{-1} \cdot \mathrm{m}^{-2}\right)$ and PAWP (5 mmHg). 6MWD and minimum SpO2 were improved to $705 \mathrm{~m}$ and $90 \%$, respectively.

IgG4-RD is a novel clinical disease entity characterised by elevated serum IgG4 concentration and marked infiltration of lymphocytes and IgG4-positive plasma cells in various organs [1]. In this patient, the diagnosis was confirmed by a serum IgG4 level well above the cut-off value for diagnosis $\left(135 \mathrm{mg} \cdot \mathrm{dL}^{-1}\right)$ and by the typical lymphoplasmacytic infiltrates containing IgG4-positive plasma cells (>10 IgG4 cells per high-power field and an IgG4/IgG cell ratio >40\%) [5] in biopsy specimen.

INOUE et al. [6] reported two cases of IgG4-related lung disease with thickening of bronchovascular bundles and multiple centrilobular nodules on chest CT, which histologically corresponded to inflammation in the peribronchiolar area. Although our case did not show thickening of bronchovascular bundles on chest CT, other findings corresponded with these two cases. Other typical morphologic features of IgG4-RD, such as obliterative phlebitis and mild-to-moderate eosinophil infiltration, were also observed in this patient. Obliterative phlebitis is less commonly observed in IgG4-related lung diseases other than those with pseudotumour and pleuritis phenotypes [7, 8]; however, this case showed remarkable obliterative phlebitis.

IgG4-RD usually presents in the lungs with pseudotumours, interstitial pneumonia or pleuritis [3], while airway involvement is an unusual phenotype. There are a few articles available about central airway disease, such as bronchial stenosis [9] or bronchiectasis caused by direct infiltration of IgG4-positive cells around bronchovascular bundles with or without bronchiolitis $[6,7,10]$; however, we could only find two articles that focussed mainly on IgG4-related bronchiolitis [11, 12]. MATsur et al. [11] first described a case with IgG4-related bronchiolitis associated with Mikulicz's disease and autoimmune pancreatitis. Subsequently, Hasegawa et al. [12] reported a patient with IgG4-related bronchiolitis and bronchial asthma. Both of these cases were diagnosed by surgical lung biopsy and improved with steroid therapy.

IsHIDA et al. [13] reported the first case of PH associated with IgG4-RD. Although the patient underwent transbronchial lung biopsy, the $\operatorname{IgG} 4 / \operatorname{IgG}$ cell ratio was not elevated in the specimen and they were unable to prove the aetiology of $\mathrm{PH}$ histologically. Consequently, this is the first case report to provide histological evidence of $\mathrm{PH}$ associated with IgG4-related lung disease. In this case, $\mathrm{PH}$ was considered to be caused by obliterative phlebitis from IgG4-positive plasma cells, which was hemodynamically similar to pulmonary veno-occlusive disease. Pulmonary veno-occlusive disease is classified as a subgroup of pulmonary arterial hypertension with the observed post-capillary lesions of septal veins and pre-septal venules consisting of loose, fibrous remodelling of the intima that may totally occlude the lumen [14]. This case can be distinguished from pulmonary veno-occlusive disease by its completely different pathological findings.

Mediastinal lymphadenopathy or fibrosing mediastinitis, which are sometimes caused by IgG4-RD [1], can cause pulmonary arterial or venous compression [15] and should also be differentiated from obliterative phlebitis as an aetiology of PH. In this case, contrast-enhanced chest CT showed no evidence of pulmonary large vessel compression.

In conclusion, this is the first report describing histological evidence of $\mathrm{PH}$ associated with IgG4-RD, which was improved by steroid and cyclosporine therapy. Pulmonary artery catheter and lung biopsy should be considered in cases of unexplained dyspnoea and bronchiolitis. Further studies are warranted to investigate the spectrum of IgG4-RD.

@ERSpublications

Pulmonary hypertension can be caused by obliterative phlebitis in patients with IgG4-related lung disease http://ow.ly/EEjvE

Jun Fukihara ${ }^{1}$, Yasuhiro Kondoh ${ }^{1}$, Hiroyuki Taniguchi ${ }^{1}$, Tomoki Kimura ${ }^{1}$, Kensuke Kataoka ${ }^{1}$, Toshiaki Matsuda ${ }^{1}$, Toshiki Yokoyama ${ }^{1}$, Kenzo Ono ${ }^{2}$, Yukio Kashima ${ }^{3}$ and Junya Fukuoka ${ }^{3}$ 
${ }^{1}$ Dept of Respiratory Medicine and Allergy, Tosei General Hospital, Seto, Aichi, Japan. ${ }^{2}$ Dept of Pathology, Tosei General Hospital, Seto, Aichi, Japan. ${ }^{3}$ Dept of Pathology, Nagasaki University Graduate School of Biomedical Sciences, Nagasaki, Japan.

Correspondence: Yasuhiro Kondoh, Dept of Respiratory Medicine and Allergy, Tosei General Hospital 160 Nishioiwake-cho, Seto, Aichi 489-8642, Japan. E-mail: lung@tosei.or.jp

Received: July 212014 | Accepted after revision: Nov 152014 | First published online: Feb 052015

Conflict of interest: None declared.

\title{
References
}

$1 \quad$ Stone JH, Zen Y, Deshpande V. IgG4-related disease. N Engl J Med 2012; 366: 539-551.

2 Hamano H, Kawa S, Horiuchi A, et al. High serum IgG4 concentrations in patients with sclerosing pancreatitis. N Engl J Med 2001; 344: 732-738.

3 Ryu JH, Sekiguchi H, Yi ES. Pulmonary manifestations of immunoglobulin G4-related sclerosing disease. Eur Respir J 2012; 39: 180-186.

4 Watanabe N, Sakamoto K, Taniguchi $\mathrm{H}$, et al. Efficacy of combined therapy with cyclosporin and low-dose prednisolone in interstitial pneumonia associated with connective tissue disease. Respiration 2014; 87: 469-477.

5 Umehara H, Okazaki K, Masaki Y, et al. Comprehensive diagnostic criteria for IgG4-related disease (IgG4-RD), 2011. Mod Rheumatol 2012; 22: 21-30.

6 Inoue $\mathrm{D}$, Zen $\mathrm{Y}, \mathrm{Abo} \mathrm{H}$, et al. Immunoglobulin G4-related lung disease: CT findings with pathologic correlations. Radiology 2009; 251: 260-270.

7 Zen Y, Inoue D, Kitao A, et al. IgG4-related lung and pleural disease: a clinicopathologic study of 21 cases. Am J Surg Pathol 2009; 33: 1886-1893.

8 Matsui S, Hebisawa A, Sakai F, et al. Immunoglobulin G4-related lung disease: clinicoradiological and pathological features. Respirology 2013; 18: 480-487.

9 Ito $\mathrm{M}$, Yasuo $\mathrm{M}$, Yamamoto $\mathrm{H}$, et al. Central airway stenosis in a patient with autoimmune pancreatitis. Eur Respir J 2009; 33: 680-683.

10 Pifferi M, Cicco MD, Bush A, et al. Uncommon pulmonary presentation of IgG4-related disease in a 15-year-old boy. Chest 2013; 144: 669-671.

11 Matsui S, Sugiyama E, Taki H, et al. A case of bronchiolitis with IgG4-positive plasma cells infiltration associated with Mikulicz's disease and autoimmune pancreatitis. Nihon Kokyuuki Gakkai Zasshi 2009; 47: 139-144.

12 Hasegawa $\mathrm{H}$, Nakamura $\mathrm{Y}$, Kono $\mathrm{M}$, et al. Bronchiolitis with IgG4-positive plasma cell infiltration in a patient with bronchial asthma. Nihon Kokyuuki Gakkai Zasshi 2010; 48: 317-321.

13 Ishida M, Miyamura T, Sato S, et al. Pulmonary arterial hypertension associated with IgG4-related disease. Intern Med 2014; 53: 493-497.

14 Montani D, Price LC, Dorfmuller P, et al. Pulmonary veno-occlusive disease. Eur Respir J 2009; 33: 189-200.

15 Sherrick AD, Brown LR, Harms GF, et al. The radiographic findings of fibrosing mediastinitis. Chest 1994; 106: 484-489.

\section{Cystic fibrosis bone disease: is the CFTR corrector C18 an option for therapy?}

\author{
To the Editor:
}

Mutations in the gene encoding the chloride ion channel CFTR (cystic fibrosis transmembrane conductance regulator) result in cystic fibrosis (CF), the most common lethal autosomal recessive genetic disease, which causes a number of long-term health problems, such as bone disease. Osteoporosis and increased vertebral fracture risk associated with CF disease are becoming more important as the life expectancy of patients continues to improve. The aetiology of low bone density is multifactorial, and is most probably a combination of inadequate peak bone mass during puberty and increased bone loss in adults [1]. Body mass index, male sex, advanced pulmonary disease, malnutrition and chronic therapies are established additional risk factors for CF-related bone disease. In multiple studies with a large cohort of adolescent and adult CF patients, the incidence of osteopenia and osteoporosis ranges from 34\% to $79 \%$ $[1,2]$. This further translated to a 100 -fold greater risk of vertebral compression, which can decrease lung function, thus accelerating the course of the disease and decreasing the patient's quality of life.

Emerging data suggest a direct genetic component to the development of CF-related bone disease. F508del is the most common CFTR mutation, with $>80 \%$ of patients being at least heterozygous [3]. We recently 